\title{
Depressive symptoms and all-cause mortality in people with type 2 diabetes: a focus on potential mechanisms
}

\author{
Giesje Nefs, Victor J. M. Pop, Johan Denollet and François Pouwer
}

\section{Background}

Depression has been associated with increased all-cause mortality in people with type 2 diabetes.

\begin{abstract}
Aims
To test whether anhedonia, dysphoria and anxiety are differentially associated with all-cause mortality and examine symptom-specific behavioural or pathophysiological mechanisms

\section{Method}

A total of 1465 people completed the Edinburgh Postnatal Depression Scale in 2005 and were followed until death or 31 December 2010. Cox regression analyses compared survival time for people with a low $v$. high baseline dysphoria/anhedonia/anxiety score and identified mediating mechanisms.
\end{abstract}

\section{Results}

After a mean follow-up of 1878 days (s.d. =306), 139 participants had died. At all time points, people with anhedonia had an almost twofold increased mortality risk compared with those without anhedonia. Physical activity met criteria for mediation. Symptoms of dysphoria and anxiety were not associated with survival time.

\section{Conclusions}

Symptoms of anhedonia predicted shorter survival time, whereas dysphoria/anxiety did not. Mechanistic pathways, in particular physical activity, should be explored further.

\section{Declaration of interest}

None.

\section{Copyright and usage}

(c) The Royal College of Psychiatrists 2016.
The prevalence of depression (major depressive disorder or elevated depressive symptoms) is significantly higher in people with type 2 diabetes compared with those without ( $18 \%$ v. $10 \%$, odds ratio $(\mathrm{OR})=1.6,95 \%$ CI $1.2-2.0){ }^{1}$ Importantly, comorbid depression in people with diabetes not only poses a significant threat to quality of life, but is also related to other health outcomes, such as the development of microvascular complications and macrovascular disease. ${ }^{2}$ A recent meta-analysis and systematic review based on 16 longitudinal studies concluded that depression was associated with a shorter survival time in people with diabetes (hazard ratio (HR) of all-cause mortality $1.46,95 \%$ CI 1.29-1.66). ${ }^{3}$ Although based on only five studies, similar results were found for cardiovascular mortality $(\mathrm{HR}=1.39,95 \% \mathrm{CI}$ 1.11-1.73). ${ }^{3}$ In both psychiatry and cardiology, increased attention has focused on the heterogeneity of depression and its implications for health. Depression is characterised by a range of symptoms and different symptom clusters. ${ }^{4}$ Identification of symptoms, symptom clusters or subtypes of depression that are associated with the greatest risk of morbidity and mortality would facilitate both risk stratification in clinical practice and the design of more effective treatments. ${ }^{5}$ One potentially relevant distinction focuses on the two core features of depression, dysphoria (depressed mood) and anhedonia (loss of interest or pleasure). Whereas dysphoria subsumes negative emotions including feelings of sadness or emptiness, anhedonia can be conceptualised as a condition in which positive affect is reduced. ${ }^{6}$ Anhedonia - or low positive affect - has been shown to predict cardiovascular conditions and mortality in community-dwelling elderly people and those with established heart disease, even after taking the presence of negative emotions into account. ${ }^{7-12}$ Similar results for all-cause mortality have been found in people with diabetes using data from the National Health and Nutrition Examination Study I Epidemiologic Follow-Up Study, ${ }^{13}$ but this study did not specify the type of diabetes participants had. The behavioural or pathophysiological pathways through which depression increases the risk of all-cause mortality remain unclear. Candidate mechanisms include suboptimal self-care behaviours and other (cardio)vascular risk factors such as obesity, hyperglycaemia, hypertension and high cholesterol. ${ }^{14}$ Whether these risk mechanisms are linked to specific depression symptoms in people with type 2 diabetes is yet to be determined. The aim of the present study was to test whether symptoms related to the two core features of depression - dysphoria and anhedonia - were differentially associated with all-cause mortality in a large cohort of people with type 2 diabetes from The Netherlands. Knowing that anxiety symptoms may figure prominently in depression and appear to adversely affect patient outcomes, ${ }^{15}$ anxiety symptoms were included in the analyses as another measure of negative affect. No study has longitudinally examined the relationship between anxiety and mortality in people with type 2 diabetes, but previous work in other clinical samples and the general population suggests that anxiety can either operate as a health-promoting or a health-compromising factor. ${ }^{16,17}$ As a second aim, in case of a significant association between specific depressive symptoms and all-cause mortality, we sought to determine which behavioural or pathophysiological mechanisms might be involved in mediating any symptom-specific association(s) with mortality.

\section{Method}

The DiaDDZoB (Diabetes, Depression, Type D personality Zuidoost-Brabant) Study was designed as a prospective cohort study among people with type 2 diabetes from primary care practices in South-East Brabant, The Netherlands, aiming to examine the course and health consequences of emotional distress in this group. $^{18} \mathrm{~A}$ total of 2460 individuals $(82 \%$ of those considered for study inclusion) participated in the 2005 baseline assessment, consisting of a nurse-led interview and the completion of a self-report questionnaire. To increase the accuracy of 
mortality records, record linkage was sought with the PHARMO Database Network, ${ }^{19}$ a population-based patient-centric data tracking system that started in 1986 that includes high-quality and complete information of patient demographics, drug dispensing, admissions to hospital, clinical laboratory, pathology and general practitioner information about 3.2 million community-dwelling inhabitants of 65 municipal areas in The Netherlands. As both the DiaDDZoB and PHARMO databases only contain de-identified patient information, record linkage was realised based on the combination of date of birth, gender, first initial, first letter of family name, first letter of marital name (women only) and zip code. This procedure resulted in successful record linkage for $81 \%(n=1982)$ of the original DiaDDZoB cohort.

The present sample $(n=1465)$ includes all DiaDDZoB participants for whom linkage with the PHARMO Database Network could be realised and who completed at least nine items on the Edinburgh Postnatal Depression Scale (EPDS) ${ }^{20}$ during the 2005 baseline assessment. Follow-up extended to either date of death (end-point) or the end of the study period (31 December 2010). The DiaDDZoB study protocol was approved by the medical research ethics committee of a local hospital, the Máxima Medical Centre in Veldhoven (NL27239.015.09). Written informed consent was obtained from all participants. The PHARMO compliance committee gave permission to establish the link between the DiaDDZoB cohort and the PHARMO Database Network.

\section{Assessment of depressive symptoms}

Presence of symptoms related to the two key elements of depression (dysphoria, anhedonia) and additional symptoms of anxiety during the past 7 days were assessed using a validated Dutch version of the EPDS. ${ }^{20}$ Originally designed to assess postpartum depressive symptoms, this questionnaire has now been validated in several other male and female samples, including people with type 2 diabetes in primary care. ${ }^{21}$ Total EPDS scores are determined by summing the scores of all ten individual items (four-point scale for each item, total score range 0-30), with higher scores indicating higher levels of depressive symptoms. In the present study, total EPDS scores were only calculated when at least nine items were completed, replacing the missing value with the mean of the remaining items. In previous research, three different EPDS symptom dimensions (factors) have been identified: depressed mood or dysphoria (four items, such as 'I have felt sad or miserable'), anhedonia (two items, such as 'I have looked forward with enjoyment to things') and anxiety (three items, such as 'I have been anxious or worried for no good reason'). ${ }^{22,23}$ In a previous paper based on 5772 people with type 2 diabetes from the same catchment area, a confirmatory factor analysis confirmed adequate fit of the three-factor EPDS structure (dysphoria, anhedonia, anxiety) to our own data, with $\chi^{2}(24)=229$, $P<0.001$ (likely because of the large sample size), root mean square error of approximation (RMSEA) 0.04 (90\% CI $0.03-$ 0.04), comparative fit index (CFI) $=0.99$ and Tucker-Lewis Index $(\mathrm{TLI})=0.98 .^{23}$ In the present study, Cronbach's alpha for the subscales/factors were $0.72,0.81$ and 0.66 respectively. As the cut-off of the ten-item EPDS (usually $\geqslant 12$ ) generally corresponds to the upper 90th percentile score, high scores on its subscales/ factors were also defined using the 90th percentile cut-off $\left(\right.$ dysphoria $\geqslant 4$, anhedonia $\geqslant 3$, anxiety $\geqslant 6$ ). ${ }^{23}$

\section{All-cause mortality}

Date of death was established using the PHARMO Database Network and records provided by general practitioners/primary care practice nurses participating in the DiaDDZoB Study.

\section{Baseline demographics, medical history, clinical values and health behaviours}

Information regarding gender, age, ethnicity ((White) Western $v$. (minority ethnic) non-Western), educational level (middle/high $v$. low), marital status (having a partner $v$. being single) and diabetes duration (less than 3 years $v .3$ years or more) was obtained during an interview with the person with diabetes by the primary care practice nurse or was part of the questionnaire booklet that had to be filled in at home by participants. The primary care practice nurse took a medical history, after which all self-reported medical diagnoses were verified through inspection of the medical record. Hyperglycaemia treatment was determined from dispensing records in the 6-month period leading up to the baseline assessment, using the community pharmacy drug dispensing records from the PHARMO Database Network. The Diagnostic Centre Eindhoven, a primary care diagnostic institute, provided results from standard care laboratory tests (fasting glucose, haemoglobin $\mathrm{A}_{1 \mathrm{c}}\left(\mathrm{HbA}_{1 \mathrm{c}}\right)$, cholesterol and albumin levels) and physical examinations (blood pressure, body mass index, eye screening). The results from yearly digital fundus photography were available to ascertain retinopathy (no/yes) and albumin level in a random urine sample was used as a proxy for nephropathy. Micro- and macroalbuminuria were defined as urine albumin concentrations $20-200$ and $>200 \mathrm{mg} / \mathrm{L}$ respectively. Additional medical comorbidities included cardiovascular disease (myocardial infarction, bypass/angioplasty, stroke and/or arterial disease) and other chronic conditions (kidney disease, asthma/ chronic obstructive pulmonary disease, cancer, arthrosis and/or rheumatoid arthritis). Health behaviours included smoking (no $v$. one or more cigarettes/day), alcohol intake ( $\leqslant 14$ v. $>14$ drinks/week), and physical activity, defined by hours of 'active' (daily activities including gardening, walking, climbing stairs) and 'sportive' activities (such as sports, fitness) per week.

\section{Statistical analyses}

Survival time was visualised by means of Kaplan-Meier curves, using the log-rank test to compare the two survival curves for participants scoring low $v$. high on (a) dysphoria, (b) anhedonia and (c) anxiety. A univariable Cox regression analysis was conducted to calculate an effect size for the association between the depressive symptom factor scores and survival time, reporting the hazard ratio with corresponding $95 \%$ confidence interval. The proportional hazards assumption was checked by visual inspection of the Kaplan-Meier survival curves (using Cox regression with a time-dependent covariate only when these suggested violation), and the Harrel and Lee test based on the Schoenfeld residuals. ${ }^{24}$ To evaluate whether the association between depressive symptom factor scores and all-cause mortality was confounded by specific demographic or clinical factors, we calculated the percentage change in the regression coefficient for the EPDS subscale before and after adjustment for individual candidate confounders. ${ }^{24}$ All variables resulting in a more than $10 \%$ change in the regression coefficient were considered to be meaningful confounders ${ }^{24}$ and were included in a multivariable-adjusted model. This approach to confounder selection was chosen a priori over inclusion of all demographic and clinical factors, as the interpretation of the 'true' association between an EPDS subscale and mortality may be improved by only including meaningful confounding factors in the multivariable model.

For all associations that became/remained significant after taking confounding factors into account, potential pathophysiological and behavioural mediators were examined. To establish (plausible) mediation, candidate mediators had to meet the four mediation criteria formulated by Baron \& Kenny. ${ }^{25}$ To this end, a series of 
linear, logistic and Cox regression analyses were conducted. In a first step, the independent variable had to be associated with the mediator (regressing the candidate mediator on the EPDS subscale). Second, the independent variable had to be associated with the dependent variable (as was done in the previously mentioned univariable Cox regression analysis, by regressing time to event on the EPDS subscale). Third, the mediator had to be associated with the dependent variable, after adjusting for the independent variable (regressing time to event on both the mediator and the EPDS subscale). Finally, the effect of the independent variable on the dependent variable in step three had to be smaller than in step two. When a candidate variable met all four criteria, we evaluated the plausibility of the mediator by examining the magnitude of the change in a last step. To do so, we determined the percentage change in the effect size (HR) of the association between the EPDS subscale and time to event before and after adjustment for the potential mediator. ${ }^{26}$ If a variable resulted in a more than $5 \%$ change $^{26}$ and fulfilled all four criteria by Baron \& Kenny, ${ }^{25}$ it was marked as a plausible mediator. All analyses were performed using PASW Statistics version 19 (IBM SPSS Statistics, Somers, New York, USA). A $P<0.05$ was considered to be statistically significant.

\section{Results}

Baseline demographics, clinical factors and health behaviours of the total sample $(n=1465)$ are shown in Table 1 . The sample for the present study did not differ significantly from the rest of the DiaDDZoB cohort $(n=995)$ with respect to gender, age, educational level, the presence of microvascular complications, diabetes duration, $\mathrm{HbA}_{1 \mathrm{c}}$ and fasting glucose, body mass index, high-density cholesterol, systolic and diastolic blood pressure, time spent in activities requiring mild to moderate physical activity or alcohol consumption. However, they were somewhat more likely to engage in sportive physical activity $(32 \% v$ v. $28 \%$, $P=0.03)$ and less likely to have non-Western ethnicity (2\% v. $5 \%, P=0.001)$, to be single ( $24 \%$ v. $30 \%, P=0.02)$, to smoke (14\% v. $19 \%, P=0.003)$, to have kidney disease (3\% v. $5 \%$, $P=0.047)$ or to have a history of cardiovascular disease $(33 \% v$. $39 \%, P=0.003)$, in particular arterial disease $(21 \%$ v. $28 \%$, $P<0.001)$. In addition, they had slightly lower mean levels of total cholesterol $(4.5$ v. $4.7 \mathrm{mmol} / \mathrm{L}, P=0.003)$ and low-density cholesterol ( 2.6 v. $2.7 \mathrm{mmol} / \mathrm{L}, P=0.006)$.

All depressive symptom factor scores were significantly correlated, with Pearson's $r=0.54$ for dysphoria and anhedonia, 0.59 for dysphoria and anxiety and 0.32 for anhedonia and anxiety (all $P<0.001$ ). A high dysphoria score was present in $13 \%$ $(n=188)$, anhedonia in $12 \%(n=170)$ and anxiety in $10 \%$ $(n=152)$ of the participants. Although the majority reported symptoms from only one category $(n=211), 131$ individuals experienced symptoms from two or three factors.

More specifically, 65 people only had a high dysphoria score, 82 people only had a high anhedonia score, 64 people only

\begin{tabular}{|c|c|}
\hline \multicolumn{2}{|l|}{ Characteristic } \\
\hline \multicolumn{2}{|l|}{ Demographics } \\
\hline Female, $n(\%)$ & $743(51)$ \\
\hline Age, years: mean (s.d.) & $67(10)$ \\
\hline Non-Western ethnicity, $n$ (\%) & $32(2)$ \\
\hline Low educational level, $n$ (\%) & $889(64)$ \\
\hline Being single, $n(\%)$ & $342(24)$ \\
\hline \multicolumn{2}{|l|}{ Medical history, n (\%) } \\
\hline Cardiovascular disease & 478 (33) \\
\hline Myocardial infarction & $160(11)$ \\
\hline Bypass/angioplasty & $190(13)$ \\
\hline Stroke & $97(7)$ \\
\hline Arterial disease & 306 (21) \\
\hline Microvascular disease & 358 (33) \\
\hline Retinopathy & $54(5)$ \\
\hline Micro- and/or macroalbuminuria & $321(25)$ \\
\hline Other chronic conditions & $699(48)$ \\
\hline Kidney disease & 49 (3) \\
\hline Asthma/chronic obstructive pulmonary disease & $177(12)$ \\
\hline Cancer & $133(9)$ \\
\hline Arthrosis & 466 (32) \\
\hline Rheumatoid arthritis & $102(7)$ \\
\hline \multicolumn{2}{|l|}{ Clinical values } \\
\hline \multicolumn{2}{|l|}{ Hyperglycaemia treatment, $n$ (\%) } \\
\hline Lifestyle only & $368(25)$ \\
\hline Oral agents, without insulin & $1021(70)$ \\
\hline Insulin (with or without oral agents) & $76(5)$ \\
\hline Diabetes duration $\geqslant 3$ years, $n(\%)$ & $870(60)$ \\
\hline Haemoglobin $A_{1 c} \%$ [mmol/mol]: mean (s.d.) & $6.7(0.8)[50(9)]$ \\
\hline Fasting glucose, mmol/L: mean (s.d.) & $7.1(1.6)$ \\
\hline Body mass index, kg/m²: mean (s.d.) & $28.8(4.7)$ \\
\hline Total cholesterol, mmol/L: mean (s.d.) & $4.5(1.0)$ \\
\hline Low-density cholesterol, mmol/L: mean (s.d.) & $2.6(0.8)$ \\
\hline High-density cholesterol, mmol/L: mean (s.d.) & $1.2(0.4)$ \\
\hline Systolic blood pressure, mmHg: mean (s.d.) & $141.7(18.4)$ \\
\hline Diastolic blood pressure, mmHg: mean (s.d.) & $78.1(9.5)$ \\
\hline \multicolumn{2}{|l|}{ Health behaviours, $n$ (\%) } \\
\hline 'Active' physical activity & $1237(85)$ \\
\hline 'Sportive' physical activity & $465(32)$ \\
\hline Current smoking & $211(14)$ \\
\hline Alcohol consumption & $116(8)$ \\
\hline
\end{tabular}

had a high anxiety score, 43 reported both dysphoria and anhedonia, 43 reported both dysphoria and anxiety, 8 reported both anhedonia and anxiety, and 37 people reported high scores on all three factors. This means that only $29 \%(n=80)$ of participants with a high dysphoria and/or anhedonia score $(n=278)$ reported both high dysphoria and anhedonia. At the end of the 5.5-year study period (mean follow-up 1878 days (s.d.=306), range 26-1964), 139 participants had died. People with a high anhedonia score were significantly more likely to have died, compared with their counterparts with low anhedonia symptoms (Table 2). Anhedonia remained significantly associated with increased odds of all-cause mortality after adjustment for symptoms of dysphoria and anxiety, gender, age

Table 2 Number of deaths (all-cause) during follow-up, stratified by dysphoria, anhedonia and anxiety

\begin{tabular}{|c|c|c|c|c|c|}
\hline & \multicolumn{3}{|c|}{ Deaths, \% $(n / N)$} & \multirow[b]{2}{*}{ Unadjusted OR (95\% Cl) } & \multirow[b]{2}{*}{ Adjusted $^{\mathrm{a}}$ OR $(95 \% \mathrm{Cl})$} \\
\hline & Low EPDS subscale score & High EPDS subscale score & $P$ & & \\
\hline Dysphoria & $9(117 / 1277)$ & $12(22 / 188)$ & 0.27 & $1.31(0.81-2.13)$ & $0.97(0.52-1.80)$ \\
\hline Anhedonia & $9(113 / 1295)$ & $15(26 / 170)$ & 0.006 & $1.89(1.19-2.99)$ & $1.84(1.07-3.17)$ \\
\hline Anxiety & $9(120 / 1313)$ & $13(19 / 152)$ & 0.18 & $1.42(0.85-2.38)$ & $1.51(0.81-2.82)$ \\
\hline
\end{tabular}




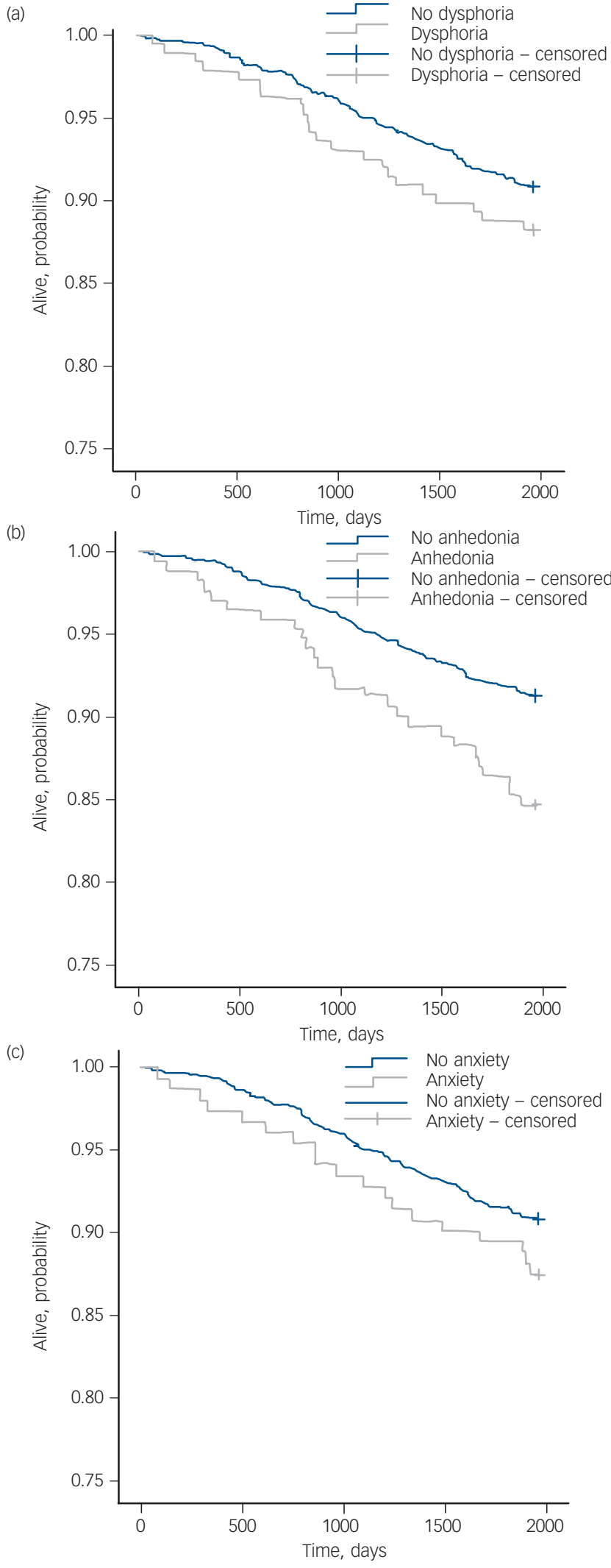

Fig. 1 Kaplan-Meier curves all-cause mortality for low v. high (a) dysphoria, (b) anhedonia and (c) anxiety.

(a) Dysphoria: log-rank test $\chi^{2}(1)=1.32, P=0.25$. No dysphoria mean days $1883(95 \%$ Cl 1867-1899); dysphoria mean days 1845 (95\% Cl 1793-1898); unadjusted hazard ratio (HR) $=1.31$ (95\% Cl 0.83-2.06). (b) Anhedonia: log-rank test $\chi^{2}(1)=7.79, P=0.005$ No anhedonia mean days 1886 (95\% Cl 1870-1901); anhedonia mean days 1823 (95\% Cl 1763-1882); unadjusted HR $=1.82$ (95\% Cl 1.19-2.79). (C) Anxiety: log-rank test $\chi^{2}(1)=1.86, P=0.17$. No anxiety mean days $1882(95 \% \mathrm{Cl} 1866-1898)$; anxiety mean days 1845 (95\% Cl 1785-1904); unadjusted HR=1.40 (95\% Cl 0.86-2.27). and cardiovascular disease history. Symptoms of dysphoria and anxiety were not associated with all-cause mortality in univariable or multivariable analyses.

After inspection of the Kaplan-Meier curves for all-cause mortality (Fig. 1) and examination of the correlation between the Schoenfeld residuals and ranked survival time (with $r$ ranging between -0.09 and -0.009 , all non-significant), the proportional hazards assumption was verified for all three symptom factors. The survival curves for participants scoring low $v$. high on anhedonia differed significantly (log-rank test $\chi^{2}(1)=7.79$, $P=0.005$ ), where people with a high score had a shorter mean time to event (1823 days, 95\% CI 1763-1882) than those with low anhedonia scores (1886 days, 95\% CI 1870-1901, Fig. 1(b)).

At any given time during the follow-up period, people with symptoms of anhedonia had a 1.8-fold increased risk of dying compared with their counterparts without anhedonia (95\% CI 1.19-2.79). Anhedonia remained a significant predictor of survival time $(\mathrm{HR}=1.78,95 \% \mathrm{CI} 1.11-2.84, P=0.02)$, after adjustment for symptoms of anxiety $(\mathrm{HR}=1.26,95 \% \mathrm{CI} 0.74-2.15, P=0.40)$ and dysphoria ( $\mathrm{HR}=0.96,95 \% \mathrm{CI} 0.56-1.64, P=0.87)$. Gender, age and marital status were identified as meaningful confounders (Table 3) and adjusted for in the multivariable model, but this attenuated the HR for anhedonia only slightly ( $\mathrm{HR}=1.72,95 \%$ CI 1.11-2.65, $P=0.02$ ). In the multivariable models, no significant association was found for anxiety $(\mathrm{HR}=1.44,95 \%$ CI $0.83-2.51$, $P=0.20)$ or dysphoria (HR $=0.61,95 \%$ CI $0.30-1.24, P=0.17$ ).

Regarding the association between anhedonia and all-cause mortality, both measures of physical activity met the criteria for mediation. Whereas active physical activity changed the strength of the association between anhedonia and survival time by $23 \%$ (adjusted HR $=1.42$, 95\% CI $0.92-2.20, P=0.12$ ), adjustment for sportive physical activity only resulted in a $6 \%$ lower and still significant HR for anhedonia (1.77, 95\% CI 1.15-2.71, $P=0.009)$. Repeating the mediation analysis for the confounder-adjusted anhedonia model resulted in a $19 \%$ reduction in effect size when active physical activity was added to the model ( $\mathrm{HR}=1.41,95 \%$ CI $0.90-2.20, P=0.14$ ).

\section{Discussion}

\section{Key findings and interpretation}

In a sample of 1465 people with type 2 diabetes, symptoms with an emphasis on reduced positive affect (anhedonia) predicted all-cause mortality over a period of 5.5 years, whereas symptoms focusing on negative emotions (dysphoria, anxiety) did not. Even after adjustment for demographic and clinical confounders, individuals with high levels of anhedonia still had an almost twofold increased risk of dying from all causes at any moment during follow-up compared with their counterparts with low levels of anhedonia. A low level of physical activity (particularly active physical activity, relating to daily activities of mild to moderate intensity) was identified as a plausible mediating mechanism in the association between anhedonia and survival time.

Similar relationships with all-cause mortality have been found in a community sample of older adults ${ }^{8}$ and people with diabetes $^{13}$ even after adjustment for negative emotions. A population-based study among older adults also found an inverse association between positive affect and the incidence of stroke. ${ }^{7}$ Research in people with established cardiovascular disease also suggests an association of anhedonia with adverse clinical outcomes. $^{9-11}$ However, as these studies have used composite end-points including both mortality and major adverse cardiac events, it is hard to disentangle whether the association with 


\begin{tabular}{|c|c|c|c|}
\hline & Dysphoria (dichotomous) & Anhedonia (dichotomous) & Anxiety (dichotomous) \\
\hline \multicolumn{4}{|l|}{ Demographics } \\
\hline Female & +32.6 & +10.9 & +34.0 \\
\hline Age, years & +3.7 & -10.0 & +11.9 \\
\hline Low educational level & -24.8 & -7.6 & -15.0 \\
\hline Being single & -41.9 & -13.6 & -1.1 \\
\hline \multicolumn{4}{|l|}{ Medical history } \\
\hline Cardiovascular disease & -11.2 & -8.3 & +7.4 \\
\hline Microvascular disease & -34.9 & -5.7 & +6.3 \\
\hline Other chronic conditions & -33.6 & -4.8 & -9.8 \\
\hline Diabetes duration $\geqslant 3$ years & -8.3 & -8.0 & +3.3 \\
\hline Dysphoria & - & -2.3 & -22.1 \\
\hline Anhedonia & -86.1 & - & -36.4 \\
\hline Anxiety & -37.1 & -6.5 & - \\
\hline
\end{tabular}

anhedonia holds for mortality only or also for the development of cardiovascular disease in these groups.

In the present study, we had no data on the cause of death. Premature cardiovascular and microvascular conditions represent the most common cause of morbidity and mortality in people with diabetes, but diabetes is associated with a higher risk of death for many diseases, including several specific forms of cancer. ${ }^{27}$ Likewise, depression is a risk factor for all major disease-related causes of death. ${ }^{28}$ Whereas depression has been associated with cardiovascular mortality in people with diabetes in some but not all studies, ${ }^{3}$ it may also play a role in deaths not caused by cancer or atherosclerotic cardiovascular disease. ${ }^{29}$

The association between anhedonia and subsequent all-cause mortality was partly explained by a low level of physical activity, in particular relating to daily activities of mild to moderate intensity. Interestingly, none of the pathophysiological cardiovascular risk factors appeared to play a mediating role. Likewise, in the Heart and Soul Study, a cohort of more than 1000 people with stable coronary heart disease, the association between positive affect and improved survival was also partly explained by physical activity, whereas a range of pathophysiological factors (heart rate variability, noradrenaline and cortisol excretion, serotonin levels, C-reactive protein level and levels of omega-3 fatty acids) only accounted for a small or negligible part of the association. ${ }^{30}$
Although mediation was not directly tested, the association between depression and adverse cardiovascular events - including deaths because of coronary, cerebrovascular or peripheral arterial disease - in people with diabetes was somewhat attenuated after simultaneous addition of body mass index, smoking, limited physical activity and $\mathrm{HbA}_{1 \mathrm{c}}{ }^{2}$ Although these factors appeared to be particularly important in cardiovascular-related mortality among people with diabetes, they did not fully explain the association with all-cause mortality. ${ }^{29}$ However, the health benefits of physical activity are well known. ${ }^{31}$ A recent Taiwanese 13 -year prospective cohort study among almost 500000 individuals showed that even people who exercise only $15 \mathrm{~min}$ a day have a $14 \%$ reduced risk of all-cause mortality and a 3-year longer life expectancy than those who are inactive. ${ }^{31}$ Furthermore, every additional $15 \mathrm{~min}$ of daily exercise further reduced mortality by $4 \% .^{31}$ Anhedonia has been associated with significantly lower levels of mild, moderate and vigorous physical activity, ${ }^{23,32}$ which appears to stem at least in part from diminished feelings of reward in response to physical activity in individuals with anhedonia. ${ }^{32}$

Other potential mediating mechanisms - not necessarily restricted to cardiovascular disease - that were not addressed in the present study may also underlie the link between anhedonia and mortality in diabetes. By definition, people with anhedonia display decreased interest and pleasure (DSM-IV-TR), ${ }^{33}$ elements

Table 4 Criteria 1 and 3 of the mediation model, and per cent change in the strength of the association (hazard ratio, HR) between anhedonia and all-cause mortality, after adjustment for potential mediators

\begin{tabular}{|c|c|c|c|c|c|c|c|c|c|c|}
\hline & \multicolumn{3}{|c|}{ Step $1^{a}$} & \multicolumn{2}{|l|}{ Step $3^{b}$} & \multicolumn{2}{|c|}{ Before adjustment } & \multicolumn{2}{|c|}{ After adjustment } & \multirow{2}{*}{$\begin{array}{l}\text { Change } \\
\text { in } \mathrm{HR}, \%\end{array}$} \\
\hline & OR (95\% Cl) & $B$ & $P$ & HR $(95 \% \mathrm{Cl})$ & $P$ & $\mathrm{HR}(95 \% \mathrm{Cl})$ & $P$ & $\mathrm{HR}(95 \% \mathrm{Cl})$ & $P$ & \\
\hline Alcohol intake & $0.79(0.42-1.50)$ & & 0.47 & $0.91(0.48-1.74)$ & 0.78 & $1.848(1.206-2.832)$ & 0.005 & $1.846(1.204-2.828)$ & 0.005 & -0.1 \\
\hline Smoking & $1.52(1.01-2.29)$ & & 0.047 & $1.47(0.97-2.22)$ & 0.07 & $1.826(1.192-2.797)$ & 0.006 & $1.777(1.158-2.725)$ & 0.008 & -2.7 \\
\hline 'Active' physical activity & $0.31(0.22-0.45)$ & & $<0.001$ & $0.33(0.23-0.47)$ & $<0.001$ & $11.842(1.203-2.823)$ & 0.005 & $1.422(0.918-2.202)$ & 0.12 & -22.8 \\
\hline 'Sportive' physical activity & $0.66(0.45-0.95)$ & & 0.03 & $0.40(0.26-0.64)$ & $<0.001$ & $11.878(1.225-2.879)$ & 0.004 & $1.767(1.152-2.712)$ & 0.009 & -5.9 \\
\hline Body mass index & & -0.03 & 0.93 & $0.95(0.91-0.99)$ & 0.01 & $1.571(0.972-2.539)$ & 0.07 & $1.551(0.959-2.508)$ & 0.07 & -1.3 \\
\hline Fasting glucose & & 0.14 & 0.27 & $0.78(0.68-0.88)$ & $<0.001$ & $11.773(1.149-2.737)$ & 0.01 & $1.848(1.197-2.853)$ & 0.006 & +4.2 \\
\hline Haemoglobin $\mathrm{A}_{1 \mathrm{c}}$ & & 0.18 & 0.01 & $1.12(0.95-1.33)$ & 0.18 & $1.773(1.149-2.737)$ & 0.01 & $1.728(1.117-2.673)$ & 0.01 & -2.5 \\
\hline Total cholesterol & & 0.002 & 0.98 & $0.89(0.74-1.06)$ & 0.19 & $1.749(1.114-2.745)$ & 0.02 & $1.747(1.113-2.741)$ & 0.02 & -0.1 \\
\hline High-density cholesterol & & -0.004 & 0.90 & $1.00(0.61-1.64)$ & 1.00 & $1.692(1.068-2.680)$ & 0.03 & $1.692(1.068-2.680)$ & 0.03 & 0 \\
\hline Low-density cholesterol & & -0.09 & 0.21 & $0.91(0.73-1.12)$ & 0.37 & $1.687(1.066-2.671)$ & 0.03 & $1.672(1.056-2.647)$ & 0.03 & -0.9 \\
\hline Systolic blood pressure & & -0.10 & 0.95 & $1.00(0.99-1.01)$ & 0.81 & $1.717(1.105-2.669)$ & 0.02 & $1.718(1.105-2.671)$ & 0.02 & +0.1 \\
\hline Diastolic blood pressure & & 0.32 & 0.69 & $0.96(0.94-0.98)$ & $<0.001$ & $1.717(1.105-2.669)$ & 0.02 & $1.725(1.110-2.682)$ & 0.02 & +0.5 \\
\hline
\end{tabular}


that may also tap into motivation for diabetes self-care activities other than regular physical activity, such as medication taking and eating healthy food. Anhedonia has been related to dysfunctions of the brain reward system and is usually characterised by an impaired responsiveness to rewarding stimuli. ${ }^{34}$ It may be that individuals with anhedonia are less likely to experience satisfaction or pleasure from achieving or maintaining optimal diabetes self-management, and may therefore be less likely to engage in these self-care behaviours. ${ }^{23}$ The biological correlates of positive affect are only beginning to be described, but pathophysiological pathways may include the autonomic nervous system, the hypothalamus-pituitary-adrenal axis and the immune system. ${ }^{35}$

\section{Strengths and limitations}

Apart from those already mentioned, several study limitations need to be acknowledged. First, dysphoria and anhedonia were derived from a self-report questionnaire measuring depressive symptoms and not based on a diagnostic psychiatric interview, which is considered the gold standard. Second, although reliability and structural validity of the three EPDS subscales are satisfactory, ${ }^{22,23}$ the measurement of symptom factors of dysphoria, anhedonia and anxiety is based on relatively few items. Third, although the EPDS does not include somatic symptoms of depression, we cannot rule out that somatic complaints related to diabetes have confounded responses to the cognitive-affective items. Fourth, we focused on the predictive power of baseline anhedonia and dysphoria, irrespective of the course of these symptoms. Fifth, depressive symptom factors and the potential behavioural and pathophysiological mediating factors were measured at the same point in time. Therefore, we cannot rule out the possibility that depressive symptoms were caused by these factors or that they changed over the follow-up period. For example, we cannot determine whether low physical activity was the result or cause of anhedonia. Sixth, all health behaviours, including both kinds of physical activity, were self-reported rather than objectively measured. Seventh, given the multiple statistical testing that was used in the present paper, there is an increased risk of type 1 error. However, we have tried to support a balanced interpretation of the data by not only focusing on statistical significance, but also displaying clinical significance (effect size) for the analyses. Eighth, no information was available on psychotherapeutic treatment or the prescription of pharmacological agents for depressed mood or anxiety symptoms specifically, which could have explained the null findings for dysphoria and anxiety. Finally, we studied a predominantly White Western group of people with type 2 diabetes who were treated in primary care practices and were in relatively good health. Although this group represents the majority of people currently living with diabetes in The Netherlands, it may be premature to generalise findings in this group to people with diabetes treated in secondary care or those with a migrant background. Dutch secondary care settings predominantly focus on people with type 1 diabetes or more complex type 2 diabetes, for example in people with multiple long-term vascular complications or problematic diabetes regulation, or when intensive blood glucose-lowering regimens are required. In contrast, most people with type 2 diabetes seen in primary care are in optimal glycaemic control and manage their diabetes with oral agents and lifestyle recommendations only. Furthermore, in many large Dutch cities such as Amsterdam, The Hague and Rotterdam, many people with type 2 diabetes have a migrant background (Turkish, Moroccan, Surinamese).

Strengths of the study include the large sample of people with type 2 diabetes, the longitudinal design, the innovative focus on depressive symptom factors and the examination of both behavioural and pathophysiological candidate mediators. Our study extends previous research about the association between depressive symptoms and adverse health outcomes by (a) focusing on all-cause mortality in people with type 2 diabetes; (b) including anxiety symptoms as a second measure of negative affect; (c) considering a range of potential demographic and clinical confounding factors; and (d) examining several potential behavioural and pathophysiological mediators.

\section{Clinical implications}

Although both psychotherapeutic and pharmacological intervention strategies appear to be moderately effective in reducing depressive symptoms in people with diabetes, ${ }^{36}$ compelling evidence showing that the risk of vascular events and mortality can be reduced by treating depression is currently lacking. One randomised controlled trial in older adults with diabetes and depression and treated in primary care suggested that participation in a depression management programme was associated with a decreased 5-year mortality risk (adjusted HR $=0.49,95 \%$ CI 0.24-0.98), but Thombs \& Ziegelstein have noticed several methodological limitations of this study. ${ }^{37}$ Depression intervention trials in people with established heart disease have not led to lower rates of recurrent cardiovascular events or death either. ${ }^{38}$

Our findings suggest that studying different symptoms of depression may add to a better understanding of the relationship between depression and mortality. In turn, these findings may have important implications for clinical practice. The treatment studies that have been done have not taken into account that depression is a highly heterogeneous condition and that different symptoms of depression may have diverging effects on (diabetes) prognosis. By tailoring depression treatment to an individual's predominant symptoms and related risk profile and by directly targeting the underlying (vasculo)toxic mechanisms, we might optimise our current treatment protocols and not only improve mood, but also health outcomes. For example, as the behavioural correlates of anhedonia include neglect of pleasurable avocations and social withdrawal (DSM-IV-TR), a renewal of interest and pleasure in these activities through behavioural activation may be of benefit to daily self-care behaviour as well. Established as an evidence-based treatment for depression in general, ${ }^{39}$ behavioural activation may work particularly well for individuals with pronounced symptoms of anhedonia as a result of its focus on a gradual increase of pleasant activities combined with training to overcome obstacles impeding the experience of pleasure. ${ }^{40}$ Implementation of this treatment approach in clinical practice is facilitated by the existence of detailed treatment manuals and the possibility of tailoring treatment based on a range of patient characteristics, including age, socioeconomic status, health status and comorbid conditions. ${ }^{40}$ As active physical activity appeared to play a more important role than sportive physical activity in the association between anhedonia and all-cause mortality in the present study, clinicians are advised to particularly focus on increasing daily activities of mild to moderate intensity, such as walking. The fact that only $15 \%$ of our sample did not meet criteria for adequate active physical activity suggests that - rather than taking on a large initiative in a wide group of people focusing on a small minority of inactive individuals may be the best way forward. A randomised controlled trial among people with a diagnosis of major depressive disorder with anhedonia may determine whether anhedonia-tailored treatment is more effective in reducing symptoms and improving health outcomes, and whether these effects are mediated by an increase in (active) physical activity. Because no clinical diagnoses were made 
in the present study, it remains unclear whether there was high co-occurrence of dysphoria and anhedonia in the subgroup with major depressive disorder. It is important to establish in future research whether the distinction between dysphoria and anhedonia is meaningful for people with subthreshold problems only, or also for people with moderate to severe depressive disorder.

Although studying symptoms of dysphoria and anhedonia is one promising way of examining the heterogeneity of depression, the distinction between somatic and cognitive symptoms of depression is also relevant for future research. ${ }^{5}$ A recent metaanalysis of 13 prospective studies focusing on 11128 people with heart disease found that somatic/affective symptoms were significantly associated with mortality and cardiovascular events (fully adjusted $\mathrm{HR}=1.19,95 \%$ CI $1.10-1.29$ ) but cognitive/affective symptoms were not $(\mathrm{HR}=1.04,95 \%$ CI $0.97-1.12) .{ }^{41}$

In conclusion, the present study adds to a growing body of literature demonstrating a link between anhedonia and adverse health outcomes by showing that symptoms of anhedonia were associated with shorter survival time in people with type 2 diabetes in primary care, whereas symptoms of dysphoria or anxiety were not. This association was partly explained by physical activity, particularly relating to daily activities of mild to moderate intensity. Distinguishing between different depressive symptoms may facilitate risk stratification and treatment decisions in clinical practice.

\footnotetext{
Giesje Nefs, PhD, Victor J. M. Pop, PhD, MD, Johan Denollet, PhD, François Pouwer, PhD, CoRPS - Center of Research on Psychology in Somatic diseases, Department of Medical and Clinical Psychology, Tilburg University, Tilburg, The Netherlands

Correspondence: Giesje Nefs, PhD, CORPS - Center of Research on Psychology in Somatic Diseases, Department of Medical and Clinical Psychology, Tilburg University, PO BOX 90153, 5000 LE Tilburg, The Netherlands. Email: g.m.nefs@tilburguniversity.edu

First received 6 Jun 2014, final revision 27 Mar 2015, accepted 20 Apr 2015
}

\section{Funding}

This study was supported by a ZonMW grant from The Netherlands Organisation for Health Research and Development, and by a Vici grant from The Netherlands Organisation for Scientific Research (The Hague, The Netherlands). The funding sources had no role in the design, data collection, analysis or interpretation of the study, or in the decision to submit the article for publication.

\section{Acknowledgements}

We would like to thank Colette Wijnands-van Gent (PoZOB) and Rients van Wijngaarden (PHARMO) for their assistance in data management and linkage, and Richard Holt (University of Southampton) for providing feedback on an earlier version of the manuscript.

\section{References}

1 Ali S, Stone MA, Peters JL, Davies MJ, Khunti K. The prevalence of comorbid depression in adults with type 2 diabetes: a systematic review and metaanalysis. Diabet Med 2006; 23: 1165-73.

2 Lin EH, Rutter CM, Katon W, Heckbert SR, Ciechanowski P, Oliver MM, et al. Depression and advanced complications of diabetes: a prospective cohort study. Diabetes Care 2010; 33: 264-9.

3 van Dooren FE, Nefs G, Schram MT, Verhey FR, Denollet J, Pouwer F. Depression and risk of mortality in people with diabetes mellitus: a systematic review and meta-analysis. PLoS One 2013; 8: e57058.

4 Stein DJ. Depression, anhedonia, and psychomotor symptoms: the role of dopaminergic neurocircuitry. CNS Spectr 2008; 13: 561-5.

5 Carney RM, Freedland KE. Is there a high-risk subtype of depression in patients with coronary heart disease? Curr Psychiatry Rep 2012; 14 $1-7$.
6 Nutt D, Demyttenaere K, Janka Z, Aarre T, Bourin M, Canonico PL, et al. The other face of depression, reduced positive affect: the role of catecholamines in causation and cure. J Psychopharmacol 2007; 21: 461-71.

7 Ostir GV, Markides KS, Peek MK, Goodwin JS. The association between emotional well-being and the incidence of stroke in older adults. Psychosom Med 2001; 63: 210-5.

8 Blazer DG, Hybels CF. What symptoms of depression predict mortality in community-dwelling elders? J Am Geriatr Soc 2004; 52: 2052-6.

9 Denollet J, Pedersen SS, Daemen J, de Jaegere P, Serruys PW, van Domburg RT. Reduced positive affect (anhedonia) predicts major clinical events following implantation of coronary-artery stents. J Intern Med 2008; 263: 203-11.

10 Davidson KW, Burg MM, Kronish IM, Shimbo D, Dettenborn L, Mehran R, et al. Association of anhedonia with recurrent major adverse cardiac events and mortality 1 year after acute coronary syndrome. Arch Gen Psychiatry 2010; 67: $480-8$.

11 Leroy M, Loas G, Perez-Diaz F. Anhedonia as predictor of clinical events after acute coronary syndromes: a 3-year prospective study. Compr Psychiatry 2010; 51: 8-14.

12 Damen NL, Pelle AJ, Boersma E, Serruys PW, van Domburg RT, Pedersen SS. Reduced positive affect (anhedonia) is independently associated with 7-year mortality in patients treated with percutaneous coronary intervention: results from the RESEARCH registry. Eur J Prev Cardiol 2013; 20: 127-34.

13 Moskowitz JT, Epel ES, Acree M. Positive affect uniquely predicts lower risk of mortality in people with diabetes. Health Psychol 2008; 27: S73-82.

14 Katon WJ, Lin EH, Russo J, Von Korff M, Ciechanowski P, Simon G, et al Cardiac risk factors in patients with diabetes mellitus and major depression. J Gen Intern Med 2004; 19: 1192-9.

15 Barbee J. Mixed symptoms and syndromes of anxiety and depression: diagnostic, prognostic, and etiologic issues. Ann Clin Psychiatry 1998; 10: $15-29$.

16 Mykletun A, Bjerkeset O, Overland S, Prince M, Dewey M, Stewart R. Levels of anxiety and depression as predictors of mortality: the HUNT study. Br J Psychiatry 2009; 195: 118-25.

17 Watkins LL, Koch GG, Sherwood A, Blumenthal JA, Davidson JR, O'Connor C, et al. Association of anxiety and depression with all-cause mortality in individuals with coronary heart disease. J Am Heart Assoc 2013; 2: e000068.

18 Nefs G, Pouwer F, Denollet J, Pop VJ. Psychological risk factors of microand macrovascular outcomes in primary care patients with type 2 diabetes: rationale and design of the DiaDDZoB Study. BMC Public Health 2010; 10: 388.

19 Herings RM, Bakker A, Stricker BH, Nap G. Pharmaco-morbidity linkage: a feasibility study comparing morbidity in two pharmacy based exposure cohorts. J Epidemiol Community Health 1992; 46: 136-40.

20 Pop VJ, Komproe $\mathrm{IH}$, van Son MJ. Characteristics of the Edinburgh Post Natal Depression Scale in The Netherlands. J Affect Disord 1992; 26 105-10.

21 de Cock ES, Emons WH, Nefs G, Pop VJ, Pouwer F. Dimensionality and scale properties of the Edinburgh Depression Scale (EDS) in patients with type 2 diabetes mellitus: the DiaDDzoB study. BMC Psychiatry 2011; 11: 141.

22 Tuohy A, McVey C. Subscales measuring symptoms of non-specific depression, anhedonia, and anxiety in the Edinburgh Postnatal Depression Scale. Br J Clin Psychol 2008; 47: 153-69.

23 Nefs G, Pouwer F, Denollet J, Kramer H, Wijnands-van Gent CJ, Pop VJ. Suboptimal glycemic control in type 2 diabetes: a key role for anhedonia? J Psychiatr Res 2012; 46: 549-54.

24 Twisk JWR. Inleiding in de Toegepaste Biostatistiek [Introduction to Applied Biostatistics]. Elsevier Gezondheidszorg, 2007.

25 Baron RM, Kenny DA. The moderator-mediator variable distinction in social psychological research: conceptual, strategic, and statistical considerations. J Pers Soc Psychol 1986; 51: 1173-82.

26 Whooley MA, de Jonge P, vittinghoff E, Otte C, Moos R, Carney RM, et al. Depressive symptoms, health behaviors, and risk of cardiovascular events in patients with coronary heart disease. JAMA 2008; 300: 2379-88.

27 Campbell PT, Newton CC, Patel AV, Jacobs EJ, Gapstur SM. Diabetes and cause-specific mortality in a prospective cohort of one million U.S. adults. Diabetes Care 2012; 35: 1835-44.

28 Mykletun A, Bjerkeset O, Dewey M, Prince M, Overland S, Stewart R. Anxiety, depression, and cause-specific mortality: the HUNT study. Psychosom Med 2007; 69: 323-31.

29 Lin EH, Heckbert SR, Rutter CM, Katon WJ, Ciechanowski P, Ludman EJ, et al. Depression and increased mortality in diabetes: unexpected causes of death. Ann Fam Med 2009; 7: 414-21. 
30 Hoen PW, Denollet J, de Jonge P, Whooley MA. Positive affect and survival in patients with stable coronary heart disease: findings from the Heart and Soul Study. J Clin Psychiatry 2013; 74: 716-22.

31 Wen CP, Wai JP, Tsai MK, Yang YC, Cheng TY, Lee MC, et al. Minimum amount of physical activity for reduced mortality and extended life expectancy: a prospective cohort study. Lancet 2011; 378: 1244-53.

32 Leventhal AM. Relations between anhedonia and physical activity. Am J Health Behav 2012; 36: 860-72.

33 American Psychiatric Association. Diagnostic and Statistical Manual of Mental Disorders (4th edn, text revision) (DSM-IV-TR). APA, 2000.

34 Hasler G, Drevets WC, Manji HK, Charney DS. Discovering endophenotypes for major depression. Neuropsychopharmacology 2004; 29: 1765-81.

35 Dockray S, Steptoe A. Positive affect and psychobiological processes. Neurosci Biobehav Rev 2010; 35: 69-75.

36 van der Feltz-Cornelis CM, Nuyen J, Stoop C, Chan J, Jacobson AM, Katon W, et al. Effect of interventions for major depressive disorder and significant depressive symptoms in patients with diabetes mellitus: a systematic review and meta-analysis. Gen Hosp Psychiatry 2010; 32: 380-95.
37 Thombs BD, Ziegelstein RC. Diabetes, depression, and death: a randomized controlled trial of a depression treatment program for older adults based in primary care (PROSPECT): response to Bogner et al. Diabetes Care 2008; 31: e54; author reply e5.

38 Pozuelo L, Tesar G, Zhang J, Penn M, Franco K, Jiang W. Depression and heart disease: what do we know, and where are we headed? Cleve Clin J Med 2009; 76: 59-70.

39 Sturmey P. Behavioral activation is an evidence-based treatment for depression. Behav Modif 2009; 33: 818-29.

40 Lejuez CW, Hopko DR, Acierno R, Daughters SB, Pagoto SL. Ten year revision of the brief behavioral activation treatment for depression: revised treatment manual. Behav Modif 2011; 35: 111-61.

41 de Miranda Azevedo R, Roest AM, Hoen PW, de Jonge P. Cognitive/affective and somatic/affective symptoms of depression in patients with heart disease and their association with cardiovascular prognosis: a meta-analysis. Psychol Med 2014; 44: 2689-703.

\section{Keeping the Woolf from the Door}

\section{Jo McFarlane}

Are you afraid of Virginia Woolf?

her crazy ramblings in the moonlit hours,

the tidal toss of mania and darkness looming like a shroud

Who's afraid of going mad?

Suicidal paranoia in a job rejection,

being sectioned for a first blow to your shadow,

coming 'home' to hospital, a four year stretch

of queuing up for liquid cosh, slippers tattered

from the endless pacing in your cubicle,

electrodes wired into your thoughts

Who's afraid?

Are you afraid of stripping like an onion,

weeping with the sting of dread

each time your name is called?

When I'm afraid

I write letters to Virginia Woolf

'What's the cleanest way to kill yourself?'

'Is it still crazy on the other side?'

She told me once there's no God in heaven,

now it's easier to pray

I wonder when my time will come

to tie a stone around my waist,

sink my sins into the river like Virginia

I'm not afraid. I'm not afraid. I'm not afraid.

Selected by Femi Oyebode. From Stigma \& Stones: Living with a Diagnosis of BPD, poems by Sally Fox \& Jo McFarlane (c) Jo McFarlane. Reprinted with permission.

Through their collection Stigma \& Stones, writers/performers/partners Sally Fox and Jo McFarlane seek to promote understanding, improve treatment and reduce the stigma of living with a diagnosis of BPD. 